\title{
Designing a Lifecycle Integrated Data Network for Remanufacturing Using RFID Technology
}

\author{
Young-woo Kim and Jinwoo Park \\ Department of Industrial Engineering/Automation System Research Institute (ASRI), \\ Seoul National University, Seoul, Republic of Korea \\ ywkim@mailab.snu.ac.kr, autofactesnu.ac.kr
}

\begin{abstract}
With the emergence of concerns regarding pollution and the exhaustion of resources, original equipment manufacturers have begun to take responsibility for environmentally sound manufacturing according to regulations that have been established. Manufacturers thus need to decide how much they will recycle and which options to pursue for minimizing operation costs and environmental impacts, while complying with regulations. They cannot, however, predict the quality of returned products, and as a result, the planning of recycling activities is not reliable. Moreover, the components of products all have different ages and lifetimes. Thus, there may be a number of components that can be recycled more than once. If the life history of these components is not available, though, recyclable components may be disposed of after being recycled once. In this paper, we propose an integrated data system that uses radio frequency identification technology to provide useful information that can make remanufacturing more efficient.
\end{abstract}

Keywords: Remanufacturing, Data System, RFID Technology, Closed-loop Supply Chain Management.

\section{Introduction}

In response to the threats of environmental pollution and the depletion of natural resources, several types of environmental legislation have come to be instituted worldwide. This legislation compels original equipment manufacturers (OEMs) to engage in environment-friendly manufacturing, based on the concept of extended producer responsibility (EPR). The term EPR, which is a preventive strategy for saving the environment, first appeared in a report of the Swedish government [1]. According to this concept, every OEM takes responsibility for the collection, recovery, and reuse of obsolete products, and performs its own disposal [2]. In addition, manufacturers must strive to implement this strategy in the design and manufacture of their products [3]. Once such environmental policies were established, a number of manufacturers invested in research on technologies that are related to recycling, such as design for disassembly [4-5].

According to Krikke (2010), the recycling of returned end-of-life (EOL) products can be classified in three ways: "product reuse," "component reuse," and "material 
recycling." under the level of disassembly [6]. It can be intuitively understood that from the standpoint of OEMs, the "product reuse" option is the most profitable approach because it requires much less additional treatment than the other options. However, the quality of returned EOL products may vary significantly according to their age and the environment in which they were used. For this reason, OEMs cannot accurately predict the quality of the returned EOL products, so their planning of recycling activities cannot be reliable.

With the development of mobile communication technologies, the use of personal electronic devices such as cellular phones has now become prevalent. The processors and components in these devices contain rare earth elements that have good stability and thermal conductivity to maximize their performance. These rare earth elements have a greater value than any other materials because they come from deposits that are smaller than the demand for them, and they are also hard to extract. In addition, about $90 \%$ of these rare earth elements are produced by the dominant producer. Hence, the recycling of personal electronic devices can be highly advantageous to OEMs from the perspective of resources and economics.

In this paper, we propose a lifecycle integrated data system for personal electronic devices that uses radio frequency identification (RFID) technology. The system assigns an exclusive identifier to every component and product to track and manage the data over the course of its entire lifecycle. This helps save natural resources by maximizing the recovery rate and save operational costs by eliminating unnecessary steps in the recovery process. It also enables manufacturers to obtain an obvious clue of recycling performance by gathering accurate information related to recycling.

\section{$2 \quad$ Related Works}

RFID technology facilitates faster and more efficient information flow and decisionmaking in supply chain management. For this reason, RFID technology has been applied in retail supply chains to streamline inventory management by providing statistical views of product shipments and inventory levels at unprecedented levels of detail [7]. It also promises to eliminate manual inventory counting, warehouse mispicking, and order numbering mistakes by providing precise data on product location, product characteristics, and product inventory levels [8]. The traceability afforded by RFID offers companies several advantages that enable them to effectively handle their daily operations with distribution transparency [9].

Based on the abovementioned advantages, the application of RFID technology to a closed-loop supply chain has also been discussed. Lee and Chan (2009) proposed an RFID-based reverse logistics framework and the optimization of the locations of collection points. These would help keep track of the quantity of returned products so as to determine the most economical transportation logistics and minimize the holding time as well as the depreciation of value [10]. Kulkarni et al. (2005) found that networked RFID systems can provide an automated and efficient approach for capturing and delivering comprehensive item-level product information in an accurate and timely manner, thereby improving both processes and decision-making during the stages 
of product recovery [11]. Nativi and Lee (2012) reported that RFID informationsharing strategies reduced inventory costs by coordinating the inventory policies of multiple decentralized players in a closed-loop supply chain [12].

The traceability afforded by RFID also facilitates lifecycle data management during the entire lifecycle of a product. Bajic and Chaxel (2002) addressed the use of automatic identification devices (Auto-ID tags) as permanent escort memories that would be associated with individual cars during their life cycle. This form of memory provides an integrated framework for vehicle lifecycle information, which is necessary to support the manufacturing, after-sales service, and recycling processes [13]. Jun et al. (2009) proposed an overall framework for RFID applications in product lifecycle management that makes use of a product-embedded information device [14]. In addition, Parlikad and McFarlane (2007) showed qualitatively that the availability of product information has a positive impact on product recovery decisions and discussed how RFID-based product identification technologies can be employed to provide the necessary information [15].

For launching RFID technology in a supply chain management system, it is essential for there to be consistent standards among the players who participate in the system. EPCglobal and GS1 are still working to develop the standards for launching RFID technology and identifying assets individually. In particular, the BRIDGE (Building Radio frequency IDentification solutions for the Global Environment) project has considered reusable assets and has defined seven identifier system keys with regard to the identifiers that are commonly employed in reusable asset management. However, they did not consider returned products, but only reusable assets such as pallets and containers [16]. Hence, alternatives for establishing standards continue to be studied because the existing standards cannot reflect the core features of recycling activities.

Luttropp and Johansson (2009) proposed the WEEE-RIM (Recycling Information Matrix) to support disassembly and recycling activities by improving the EPCglobal GID-96 standard [17]. They sought to incorporate instructions for recovery treatment into the RFID tags, which contain the following information: the company identifier, a statement as to who bears the responsibility as producer, and specific recycling information such as recycling targets, recycling actions, and hazardous materials. In this paper, we propose different ways of using external databases (DBs) based on the method proposed in their paper. We selected this approach because, in comparing these two methods, we found that the use of external DBs makes it possible to store much more detailed information than the WEEE-RIM, and that the use of the available space on the tags only for an identifier ensures that the system can identify a greater number of components.

\section{System Design}

\subsection{Overall Framework}

The information system proposed in this paper deals with data related to returned products and their components. Each piece of data will be entered in the product DB 
and the component DB when a component or a product is produced, repaired in its middle-of-life (MOL) phase, collected in its EOL phase, along with whatever other events are of interest during its entire lifecycle. These DBs would then consist of information about every product produced by the OEM, as well as every component that is included in every product family of the OEM. The details of these DBs will be presented in Section 3.3.

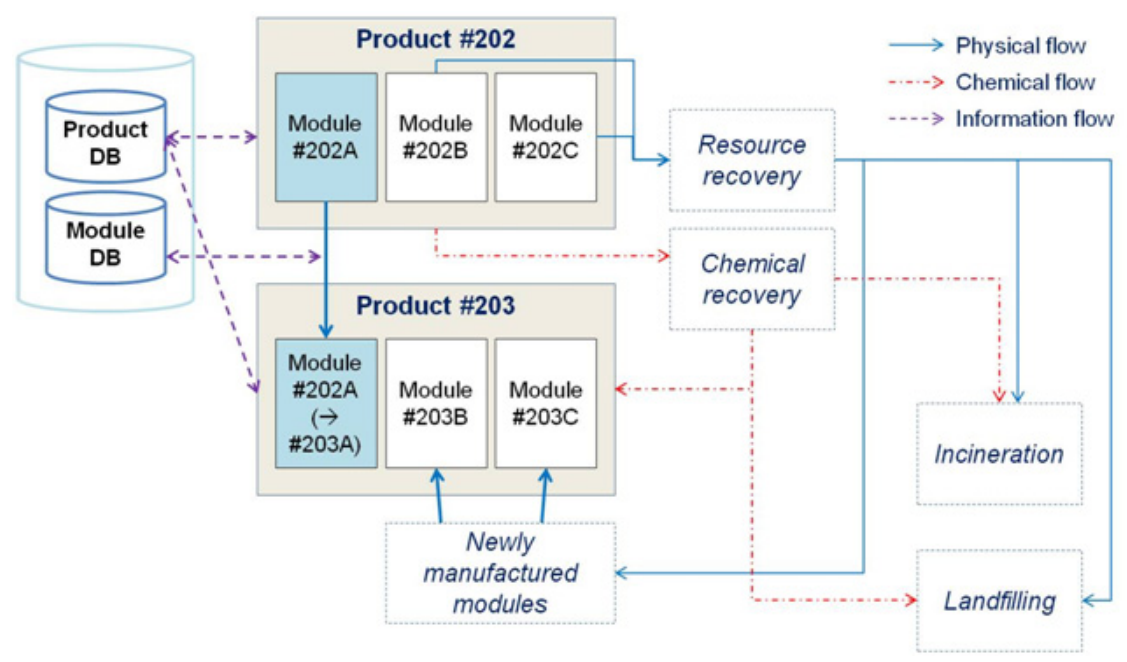

Fig. 1. Flows in remanufacturing system and recycling process

\subsection{Design of RFID Tags}

RFID technology is widely used to provide escort memory because an information system that uses RFID technology can perform the unambiguous labeling of objects by means of electronically stored data. The data identifying the object can also be read wirelessly through a radio frequency channel. A labeled object only transmits data when a matching reader initiates this process. Based on these features, RFID technology makes decision-making faster and more efficient by providing information that follows the product, by first sorting the main recycling target possibilities. We should note that in the design of tags and the corresponding DBs, the information must be coded in a systematic manner that can be understood by workers at the recycling plant. In this context, the importance of standards is an emerging issue. Fortunately, GS1, the international standards organization, has defined a standard for RFID tags called the electronic product code (EPC). EPC prescribes that a tag should contain exactly n-bits that are divided into a number of fields, where $n$ is the capacity of the tag. The EPC standards set 8 bits of space on a tag for its header, which defines the overall length, identity type, and structure of the EPC tag encoding. For example, general identifier GID-96 is defined for a 96-bit EPC as follows: The header "00110101" indicates that the tag follows the GID-96 standard, and the general manager number, object class, and serial number occupy 28, 24, and 36 bits, respectively. 


\begin{tabular}{|c|c|c|c|c|}
\hline \multicolumn{4}{c}{$n$ bits } & \multicolumn{3}{c}{} \\
\hline Header & EPR ID & REP ID & Object Class & SERIAL \\
\hline 8 bits & $a$ bits & $b$ bits & $c$ bits & $d$ bits \\
\hline
\end{tabular}

Fig. 2. Proposed design of RFID tags

In this study, we assume that n-bit tags are attached to each product and module to be identified. Thus, we divide n bits into five fields: "Header," "EPR ID," "REP ID," "Object Class," and "SERIAL." The header occupies 8 bits, which indicates that the tag is for the proposed information system. Every OEM and third-party reprocessing facility has its own exclusive ID, and this ID is the value of the 2nd field "EPR ID" and the 3rd field "REP ID." These ID fields indicate the exclusive ID of the manufacturer who is responsible for recycling and the ID of the facility that actually performs product recovery. When a manufacturer itself collects products and performs product recovery, the 2 nd and 3rd fields will have same value. The 4th field, "Object Class," indicates the type of product. The last field, "SERIAL." stores a serial number that was assigned in the manufacturing stage, so this field serves as a primary key for products and modules. By identifying products using their primary keys, we can obtain useful data from DBs about the processing of returned products at each stage. "SERIAL" is assigned when a module or product is newly manufactured. At the same time, data for the module, such as time of birth, is entered in a module DB. A product $\mathrm{DB}$, then, contains data regarding which modules compose the product.

The number of reprocessing facilities and OEMs depends on the size of the regulatory target. The total number of target OEMs and reprocessing facilities affects the total space of the RFID tags and the space that is occupied in each field, except for the header. The organization of the memory space of the tag can be flexible enough to accommodate any number of facilities and manufacturers.

As seen in Fig. 2, the proportions of storage available for "EPR ID," "REP ID," "Object Class" and "SERIAL" as well as the total capacity of the tag, can be flexibly adjusted according to a given situation in terms of the number of OEMs, facilities, and products and their modules. As a modification, we would propose that the space of the 2nd and 3rd fields be minimized as much as possible in order to assign a larger space to SERIAL. This would make the system more stable when new products and their components are introduced. It could then also deal with modules of a lower level in a bill of materials.

\subsection{Design of External Databases and Transactions}

In the beginning-of-life (BOL) phase, we assume that products contain components such as modules. The OEM starts with manufacturing subassemblies and manufacturing ends with assembly jobs. For component-level management, the OEM should record the birth history of every component as well as the information regarding which component is used for which product. The tag does not need to contain everything regarding this information, though, as it serves only as an identifier for efficient 
memory usage. All information should be stored in external DBs, and if more information about a product or component is needed, it can be accessed by sending a query with its identifier.

There are four external DBs: Product DB, Module DB, Instruction DB, and Result DB.

In the BOL phase, a product with components is manufactured. The tag to identify that product, which includes its exclusive serial number, is attached to it after the product's assembly is complete. The tag also includes information about the product and its components such as a timestamp of its birth, its object class, and its serial number, which are recorded in the product DB and module DB simultaneously. For the module DB in particular, there is a "Parent" field that indicates the component that it belongs to.

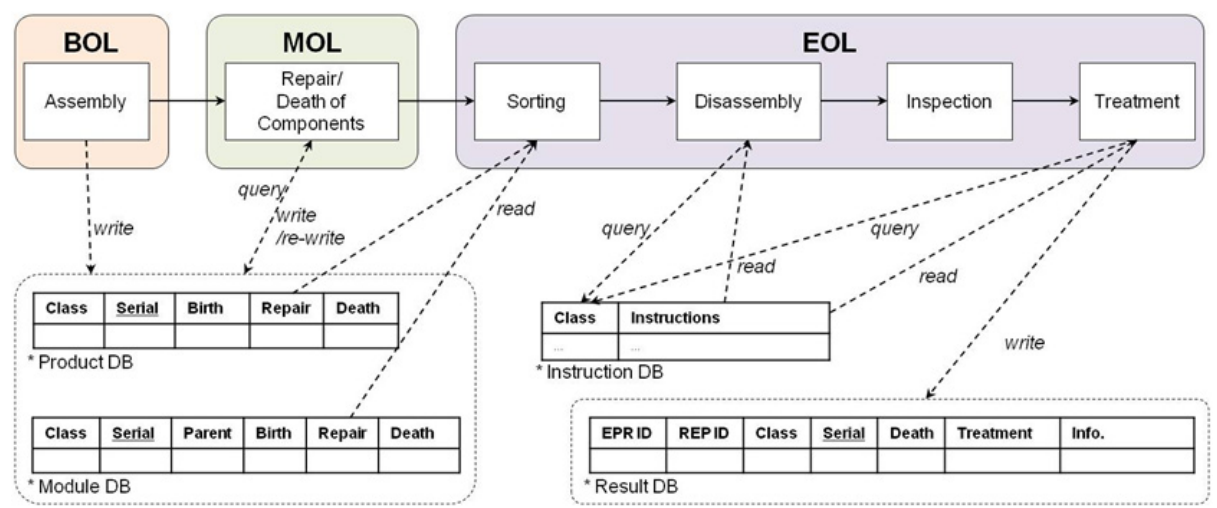

Fig. 3. Data interaction during product's entire lifecycle

In the MOL phase, component failure as well as product failure can take place. A failed component can be repaired or is considered dead if its condition renders it nonreusable. When a failed component of a product in the MOL phase is returned for repair and is replaced, the time of death should be entered in the module DB, and the product's record should be modified accordingly.

When these events take place, the timestamp of the repair or death is recorded in the product/module DB. The "Repair" field is thus an important sector in the recovery process.

In the sorting stage, the product's status that compares its expected life and its age is analyzed. If the timestamp of its repair has a null value, we can intuitively assume that the component is in good condition. If, on the other hand, the component has a repair history, the OEM should perform further inspection because the quality of the component is uncertain. This step makes the entire recovery process more efficient by omitting the additional inspection of returned products whose good quality is obvious.

Information that serves as a guide to the recycling process and as a warning to workers regarding hazardous materials can make the recycling process safer. Unlike the WEEE-RIM method, recycling information should be provided in the instruction $\mathrm{DB}$, so that workers can access the information they need for any product they 
are working with. Loading recycling information by querying an instruction DB definitely saves a greater capacity of tags better than the WEEE-RIM method.

Once the recovery process is complete, the recycling results for the modules and products, such as which option has been pursued, are recorded in the DB. Administrative organizations that manage recycling can then find data logs of the recovery process in the results DB and can then accurately evaluate their recycling performance.

\section{Conclusion}

In this paper, we proposed an RFID-based integrated data system that facilitates economically efficient and environmentally sound decision-making. RFID tags that are attached to every product and module make it possible to acquire historical information over the course of its entire lifecycle. This means that we can obtain useful data for managing the uncertainty related to a product's quality when EOL products are returned. Based on the information acquired, OEMs can determine the most appropriate recovery option to be undertaken. This system also makes it possible to establish the most profitable production plan that involves no violation of environmental regulations.

The data gathered by the RFID-based system is recorded precisely in real time. This makes it possible for administrators to perform continuous and quantitative monitoring and accurate performance measurement. The proposed system can also provide a basis for penalizing manufacturers who do not comply with regulations or reward those who have demonstrated excellent recycling performance.

The RFID tag design proposed in this paper could be used by any organization with a flexible proportion of storage capacity because we have considered the existing standard. The individual identification of products and components is based on the Object Naming Service (ONS) of EPCglobal. In this context, we need to consider the existing concept of the Discovery Service to solve the security problem that information sharing will present in the future.

In this study, we did not analyze the economic aspects related to the cost of RFID integration and the possible advantages it could offer. One aspect we would mention here, though, is that RFID tags can be used semi-permanently until the tags are broken because they are used in a closed-loop system and are rewritable. This means that once the system launched, hardly any additional costs are incurred, except for maintenance costs.

To make the proposed system helpful to manufacturers, a decision-support system should be created that evaluates the economic value of returned products and their modules, and predicts how much additional value would be generated by the different options that are available. 


\section{References}

1. Lindhqvist, T., Lidgren, K.: Model for Extended Producer Responsibility: In Ministry of the Environment, From the Cradle to the Grave - six studies of the environmental impacts of products, pp. 7-44. Ministry of Environment, Stockholm (1990)

2. Lindhqvist, T.: Extended Producer Responsibility in Cleaner Production. IIEEE Dissertatons, Lund University (2000)

3. Van Rossem, C., Tojo, N., Lindhqvist, T.: Extended producer responsibility: an examination of its impact on innovation and greening products. Greenpeace (2006), http: / / www . greenpeace.org/raw/content/international/press / reports/epr.pdf

4. Kuo, T.C.: Enhancing disassembly and recycling planning using life-cycle analysis. Robotics and Computer-Integrated Manufacturing 22, 420-428 (2006)

5. Das, K., Chowdhury, A.H.: Designing a reverse logistics network for optimal collection, recovery and quality-based product-mix planning. International Journal of Production Economics 135, 209-221 (2012)

6. Krikke, H.: Opportunistic versus life-cycle-oriented decision making in multi-loop recovery: an eco-eco study on disposed vehicles. International Journal of Life Cycle Assessment 15, 757-768 (2010)

7. Wailgum, T.: Tag, you're late: CIO Magazine, pp. 50-56 (November 15, 2004)

8. Niederman, F., Mathieu, R.G., Morley, R., Kwon, I.: Examining RFID Applications in Supply Chain Management. Communications of the ACM 50, 93-101 (2007)

9. Lee, D., Park, J.: RFID-based traceability in the supply chain. Industrial Management and Data Systems 108, 713-725 (2008)

10. Lee, C., Chan, T.: Development of RFID-based Reverse Logistics System. Expert Systems with Applications 36, 9299-9307 (2009)

11. Kulkarni, A., Parlikad, A., McFarlane, D., Harrison, M.: Networked RFID Systems in Product Recovery Management. In: IEEE International Symposium on Electronics and the Environment (2005)

12. Nativi, J., Lee, S.: Impact of RFID information-sharing strategies on a decentralized supply chain with reverse logistics operations. International Journal of Production Economics 136, 366-377 (2012)

13. Bajic, E., Chaxel, F.: Auto-ID Mobile Information System for Vehicle Life Cycle Data Management. In: IEEE Conference on Systems, Man and Cybernetics (2002)

14. Jun, H., Shin, J., Kim, Y., Kiritsis, D., Xirouchakis, P.: A Framework for RFID applications in product lifecycle management. International Journal of Computer Integrated Manufacturing 22, 595-615 (2009)

15. Parlikad, A., McFarlane, D.: RFID-based product information in end-of-life decision making. Control Engineering Practice 15, 1348-1363 (2007)

16. Bowman, P., Ng, J., Harrison, M., Illic, A.: Reusable Asset Management Model. BRIDGE Project WP3 (2009)

17. Luttropp, C., Johansson, J.: Improved Recycling with Life Cycle Information Tagged to the Product. Journal of Cleaner Production 18, 346-354 (2010) 